\title{
Evaluation of a protocol for coronary artery disease investigation in asymptomatic elderly hemodialysis patients
}

This article was published in the following Dove Press journal: International Journal of Nephrology and Renovascular Disease

\author{
Jose Jayme G De Lima' \\ Luis Henrique W Gowdak' \\ Flavio J de Paula' \\ Henrique Cotchi S Muela ${ }^{2}$ \\ Elias David-Neto' \\ Luiz A Bortolotto' \\ 'Heart Institute (InCor) and Renal \\ Transplant Unit, Division of Urology, \\ Hospital das Clínicas, University of \\ São Paulo Medical School, São Paulo, \\ SP, Brazil; ${ }^{2}$ Department of Medicine, \\ Agostinho Neto University Medical \\ School, Luanda, Angola
}

Background: Coronary artery disease (CAD) is prevalent in older patients on dialysis, but the prognostic relevance of coronary assessment in asymptomatic subjects remains undefined. We tested the usefulness of a protocol, based on clinical, invasive, and noninvasive coronary assessment, by answering these questions: Could selecting asymptomatic patients for coronary invasive assessment identify those at higher risk of events? Is CAD associated with a worse prognosis? Methods: A retrospective study including 276 asymptomatic patients at least 65 years old on the waiting list, prospectively evaluated for CAD and followed up until death or renal transplantation, were classified into two groups: 1) low-risk patients who did not undergo coronary angiography ( $\mathrm{n}=63)$ and 2 ) patients who did undergo angiography $(\mathrm{n}=213)$. The latter group was reclassified into patients with significant $\mathrm{CAD}$ or normal angiograms/nonsignificant CAD. Results: CAD ( $\geq 70 \%$ stenosis) occurred in 124 subjects (58\%). The incidence of death by any cause, coronary death, and major cardiovascular (CV) events were similar in patients selected or not for angiography and in those with or without significant CAD. Myocardial revascularization (surgical/percutaneous) was performed in only $21 / 276$ patients ( $7.6 \%$ ) and did not result in a reduction in mortality.

Conclusion: In older patients on renal replacement therapy, the prevalence of CAD was high, but coronary investigation was not useful as a risk stratification tool and also resulted in a rather small proportion of patients eligible for intervention. Therefore, in the elderly, coronary investigation should not be considered routine in asymptomatic patients.

Keywords: elderly, hemodialysis, coronary artery disease, kidney transplantation, cardiovascular, chronic kidney disease

\section{Introduction}

There were times when patients older than 60 years of age were barred from undergoing dialysis. Nowadays, a large proportion of patients starting on renal replacement therapy are older than 60 years due to a combination of factors, such as improvement in dialysis technology, increased prevalence of risk factors for renal disease, and the aging population. ${ }^{1}$ Older patients on dialysis are now surviving long enough to develop cardiovascular complications often demanding complex interventions. Because the life expectancy of these patients is relatively short, ${ }^{2}$ it is reasonable to ask whether invasive, costly, and risky procedures will translate into increased survival. There is a paucity of information on the subject, because renal disease is often a criterion for exclusion from trials dealing with the diagnosis and treatment of cardiovascular disease. For instance, in 86 cardiovascular trials that randomized more than 400,000
Correspondence: Jose Jayme G De Lima Heart Institute (InCor) and Renal Transplant Unit, Division of Urology, Hospital das Clínicas, University of São Paulo Medical School, Av Dr Enéas Carvalho de Aguiar, 44, 05403-000 São Paulo, SP, Brazil

Tel +55 II $266 \mid 5084$

Fax +55 II 266I 5948

Email jose.lima@incor.usp.br 
patients, $80 \%$ of the trials excluded subjects with end-stage renal disease, whereas baseline renal function was reported in only $7 \%$ of the trials. ${ }^{3}$

Coronary artery disease (CAD) is prevalent in patients with chronic kidney disease (CKD), especially in those undergoing dialysis. ${ }^{4}$ It is generally held that patients with symptomatic CAD must undergo coronary evaluation in accordance with guidelines developed for the general population. However, the best approach for assessment of $\mathrm{CAD}$ in asymptomatic patients with $\mathrm{CKD}$ is far from clear because, in that population, there is little correlation between the presence and severity of CAD and symptoms. ${ }^{5}$ Because $\mathrm{CAD}$ has an important impact on prognosis in patients on dialysis, ${ }^{6}$ most centers use clinical risk stratification to select patients for noninvasive and invasive coronary investigation regardless of symptoms. ${ }^{7}$ In a previous investigation, we showed that this approach is useful in a non-selected dialysis population. ${ }^{5}$ We also demonstrated that coronary angiography is the best test for assessing cardiac risk. ${ }^{6}$ However, we ignored whether this same approach would also perform well in discrete dialysis populations, like the elderly, especially in those without angina or other clear clinical manifestation of myocardial ischemia. In that group, there is no firm consensus about who should be tested, which testing modality should be used, and who should undergo intervention if CAD is found. Obviously, prospective, randomized studies devised to compare different modalities of investigation and treatments in this population are needed. Lacking such studies, it will be of interest to assess the efficacy of a protocol, based on current guidelines, using invasive and noninvasive testing in asymptomatic dialysis patients.

In 1997, we started a systematic cardiovascular evaluation, following a prespecified protocol, with the objective of defining the best approach to diagnose and treat cardiovascular disease in patients on the waiting list for renal transplantation (the KiHeart cohort) with emphasis on CAD. Since then, we have prospectively collected data from over 2,000 patients who were also followed up until death. In the present study, we retrospectively evaluated the results of the protocol in asymptomatic patients at least 65 years old, using a combination of invasive and noninvasive testing. The purpose of the study was to test the usefulness of our protocol by answering the following questions: Could selecting asymptomatic patients for invasive coronary assessment identify those at higher risk of events? Is CAD associated with a worse prognosis? Finally, we also explored the impact on prognosis of current guidelines for selecting patients for coronary intervention or clinical treatment.

\section{Patients and methods}

This work was performed in accordance with the postulates of the Helsinki Declaration, and all patients provided a signed informed consent. The Institutional Scientific Board, Heart Institute (InCor), University of São Paulo Medical School, approved the protocol. Patients were being considered for receiving their first kidney graft from a deceased donor at the Renal Transplant Unit, Division of Urology, University of São Paulo Medical School, and were referred to the Heart Institute (InCor) for cardiovascular assessment before being formally included on the waiting list. The period of inclusion ranged from January 1998 to May 2017.

A prespecified comprehensive cardiovascular investigation was performed, as reported elsewhere. ${ }^{5}$ Patients underwent a 12-lead resting electrocardiogram (EKG) and transthoracic echocardiography as part of their evaluation. Noninvasive testing for $\mathrm{CAD}$ with dipyridamole/adenosine myocardial stress testing by SPECT with ${ }^{99 m}$ Tc Sestamibi was performed, according to the protocol, independently of risk factors for CAD. The criteria to indicate coronary angiography were the following: noninvasive testing suggestive of $\mathrm{CAD}$, or an increased risk for CAD based on clinical grounds, like diabetes, stroke, left ventricular (LV) dysfunction (defined as LV ejection fraction $\leq 40 \%$ ), or peripheral vascular disease (defined as absence of lower extremity peripheral arterial pulse, previous vascular intervention, amputation, or gangrene). Significant CAD was arbitrarily defined as luminal stenosis $\geq 70 \%$ in one or more epicardial arteries or at least $50 \%$ narrowing of the main left coronary artery by visual estimation in the "worst view" angiographic projection from two independent experts.

Patients with significant CAD were evaluated by the study team, according to the American Heart Association/American College of Cardiology criteria for coronary intervention ${ }^{8}$ and selected either to continue on medical treatment or to undergo myocardial revascularization (surgical or percutaneous) plus medical treatment. Percutaneous intervention (bare metal stent) was preferred whenever the two invasive procedures were considered adequate by current guidelines. Myocardial revascularization procedures were judiciously indicated based on current guidelines and available evidence and not because patients were candidates for noncardiac surgery (kidney transplantation), because it has been shown that preoperative coronary revascularization in high-risk patients is not associated with improved outcomes. ${ }^{9}$ A detailed description of the strategy used in our center to investigate and treat $\mathrm{CKD}$ patients with $\mathrm{CAD}$ has been reported elsewhere. ${ }^{10}$

Patients had been treated by maintenance hemodialysis, for at least 3 months, performed in 4-hour sessions, 
three times/week using a bicarbonate bath. Patients were maintained on statins, aspirin, angiotensin converting enzyme inhibitors (or angiotensin receptor blockers), and $\beta$-blockers per current guidelines. ${ }^{11}$ Adherence to treatment and to a healthy lifestyle were verified and encouraged. All patients evaluated were included on the waitlist. However, the researchers had no control over the criteria used by the transplant team to withdraw patients from the list.

Censored events were determined during clinical visits, by telephone or e-mail. No individual was lost to follow-up. Patients were followed from the time of inclusion until death or renal transplantation.

The primary end point was all-cause mortality. Secondary end points were coronary death (myocardial infarction and death associated with coronary intervention) and the incidence of combined major CV events (myocardial infarction, unstable angina, peripheral vascular event, sudden death, stroke, and heart failure). Sudden death was defined as death from natural causes occurring within 1 hour after initiation of symptoms with no definitive assessment of its cause.

\section{Statistical analysis}

Values are expressed as mean, standard deviation of the mean, and percentages. The data for surgical and percutaneous interventions were pooled. For analysis of the data, we used the SPSS statistical program (SPSS Statistics, version 20.0; IBM, Armonk, NY, USA). Univariate analysis was performed with chi-squared testing, the Student $t$-test, or the median test, as indicated. Survival curves were constructed using the Kaplan-Meier method and compared using the log-rank test. Cox proportional hazards model was used to verify the variables independently associated with outcomes. The variables selected for the multivariate Cox model were age, sex, body mass index, hypertension, dyslipidemia, current/past smoking, diabetes, other cardiovascular disease, and coronary assessment. A $P$-value of less than 0.05 was considered statistically significant.

\section{Results}

Patients were selected from an original population of 2,129 patients (the KiHeart cohort). This was a single-center observational study based on data collected prospectively in 379 hemodialysis patients at least 65 years old. One-hundred three patients were excluded due to previous coronary intervention, angina, and refuse to participate or incomplete data, leaving 276 individuals for analysis (Figure 1).

Patients were divided into two groups (Figure 1): patients with a low clinical probability of CAD and normal myocardial

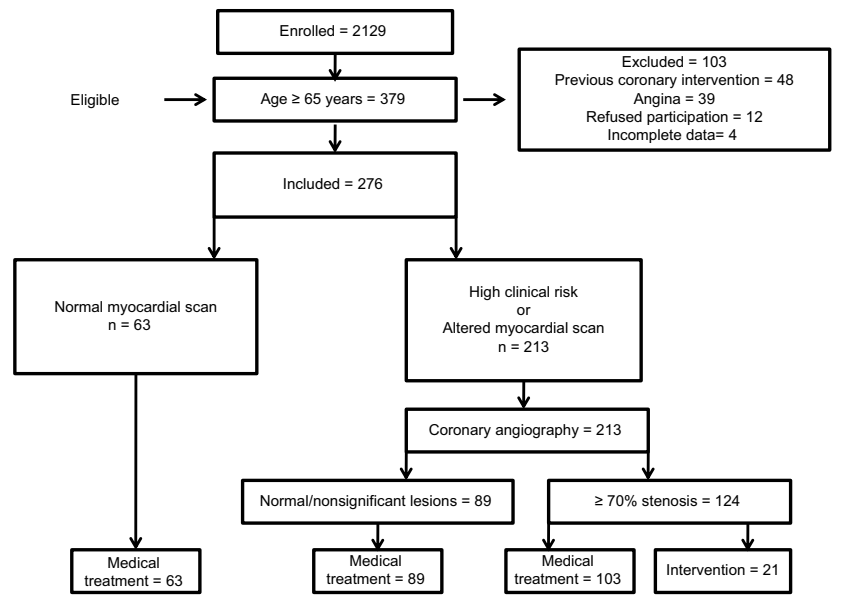

Figure I Flow chart of patients enrolled, eligible, and included in the study.

scan who did not undergo coronary angiography $(n=63)$ and patients who underwent coronary angiography because of either an abnormal myocardial scan or a history suggestive of cardiac or extra-cardiac atherosclerosis $(n=213)$. Patients pertaining to the latter group were again classified into two subgroups: those with nonsignificant coronary stenosis or normal coronary arteries $(n=89)$ who were treated medically and those with coronary stenosis $\geq 70 \%(n=124)$ who were selected either for medical treatment $(n=103)$ or intervention plus medical treatment $(n=21)$.

\section{Baseline characteristics}

Table 1 shows the baseline characteristics of patients. More than $50 \%$ of our patients had either diabetes or associated CVD or both. Patients not selected for invasive studies were slightly older, had lower body mass index, and a reduced prevalence of diabetes and associated cardiovascular disease, in part reflecting the criteria used to indicate coronary angiography. This group also included a nonsignificant reduced proportion of patients with dyslipidemia. Aspirin, betablockers, and statins were prescribed more often in patients at higher risk of CAD.

\section{Patient selection for coronary angiography and the risk of events}

Table 2 shows the causes of death. In older patients, all-cause mortality, coronary-related mortality, and the incidence of combined major $\mathrm{CV}$ events did not differ between low-risk patients and those selected for invasive coronary evaluation (Figure 2). Therefore, a higher probability of CAD, based on clinical grounds or an altered myocardial scan, was not useful as a risk stratification tool in this waiting list population. It is 
Table I Baseline characteristics in patients undergoing or not undergoing coronary angiography $(\mathrm{n}=276)$

\begin{tabular}{|c|c|c|c|}
\hline Variable & No coronary angiography, $n=63$ & Coronary angiography, $n=213$ & $P$-value \\
\hline Age, years & $70.1 \pm 3.7$ & $68.6 \pm 3.2$ & 0.006 \\
\hline Sex, male & $39(62 \%)$ & $140(66 \%)$ & 0.57 \\
\hline Race, white & $52(82 \%)$ & $157(74 \%)$ & 0.15 \\
\hline BMI, $\mathrm{kg} / \mathrm{m}^{2}$ & $24.4 \pm 3.6$ & $26.6 \pm 4.4$ & 0.0001 \\
\hline Dyslipidemia* & $13(22 \%)$ & $71(34 \%)$ & 0.077 \\
\hline Smoking** & $16(25 \%)$ & $64(30 \%)$ & 0.46 \\
\hline Diabetes & $18(29 \%)$ & $134(63 \%)$ & 0.0001 \\
\hline Hypertension & $49(78 \%)$ & $167(79 \%)$ & 0.97 \\
\hline Other CV diseases*** & $12(19 \%)$ & $118(53 \%)$ & 0.0001 \\
\hline Hematocrit, \% & $38.1 \pm 5.0$ & $37.7 \pm 5.5$ & 0.71 \\
\hline Time on dialysis, months, median & 21 & 17 & 0.55 \\
\hline \multicolumn{4}{|l|}{ Medication } \\
\hline Aspirin & $60 \%$ & $85 \%$ & 0.001 \\
\hline Beta-blockers & $47 \%$ & $77 \%$ & 0.002 \\
\hline Statins & $33 \%$ & $84 \%$ & 0.0001 \\
\hline ACEI/ARB & $25 \%$ & $52 \%$ & 0.03 \\
\hline
\end{tabular}

Notes: *Total cholesterol and/or triglycerides $\geq 200 \mathrm{mg} / \mathrm{dL} ;{ }^{* *}$ current/past ; ***myocardial infarction, stroke, peripheral arterial disease, LV systolic dysfunction. Abbreviations: BMI, body mass index; CV, cardiovascular; ACEI, angiotensin converting enzyme inhibitor; ARB, angiotensin receptor blocker; LV, left ventricular.

Table 2 Cause of death

\begin{tabular}{|l|l|}
\hline Cause of death & Patients n=276 \\
\hline Cardiovascular death & \\
\hline $\begin{array}{l}\text { Coronary } \\
\text { Myocardial infarction } \\
\text { Intervention-related }\end{array}$ & 12 \\
\hline Sudden death & 2 \\
\hline Stroke & 29 \\
\hline Heart failure & 12 \\
\hline Other CV & 10 \\
\hline Total CV & 9 \\
\hline $\begin{array}{l}\text { Non-cardiovascular death } \\
\text { Infection }\end{array}$ & $74(51.7 \%)$ \\
Malignancies & 44 \\
\hline Other non-CV & 4 \\
\hline Total non-CV & 11 \\
\hline Not determined & $59(41.3 \%)$ \\
\hline Total (CV+ non-CV+ not determined) & $10(7.0 \%)$ \\
\hline
\end{tabular}

Abbreviation: $\mathrm{CV}$, cardiovascular.

also important to note that only 14 coronary deaths occurred during an extended follow-up of more than 10 years in the totality of patients ( $9.8 \%$ of all deaths). Because coronary heart disease is a leading cause of sudden death, we repeated the analysis including sudden death, along with myocardial infarction and death associated with coronary intervention. There was no difference between groups in the incidence of coronary death $(\log$-rank $=0.532)$.

\section{Presence of CAD and the risk of events}

In 213 patients undergoing coronary angiography, CAD (stenosis $\geq 70 \%$ ) was observed in 124 patients (58\%), close to that observed in younger individuals ( $<65$ years old) from the same cohort ( $54 \%, P=0.68)$. Figure 3 shows the impact of $\mathrm{CAD}$ on the incidence of events. In patients undergoing invasive coronary assessment, the presence of CAD was not related to prognosis. Patients with and without CAD had a similar incidence of total and coronary deaths and important cardiovascular events. Again, the contribution of coronary death to overall mortality was small.

\section{Impact of coronary intervention on prognosis}

We assessed the value of current guidelines to select patients either to undergo coronary intervention plus medical treatment or medical treatment alone on prognosis. The design of this study prevents comparing medical and intervention treatments: we only assessed the consequences of applying our protocol on results. Most patients who underwent coronary angiography (192 out of $213,90 \%$, Figure 1) were treated medically. Intervention was performed in only $9.8 \%$ of individuals undergoing invasive studies or $7.6 \%$ of the totality of patients included.

Using current guidelines, in 124 subjects with CAD, myocardial revascularization (plus medical treatment) was performed in 21, whereas medical treatment alone was indicated in 103 (Figure 4). We found no significant difference in the incidence of death by any cause or in the incidence of major cardiovascular events. Coronary-related deaths tended to be increased in patients undergoing intervention. This does not necessarily mean that intervention had a negative impact on prognosis, because patients selected for intervention had, by definition, more severe CAD. Eight 

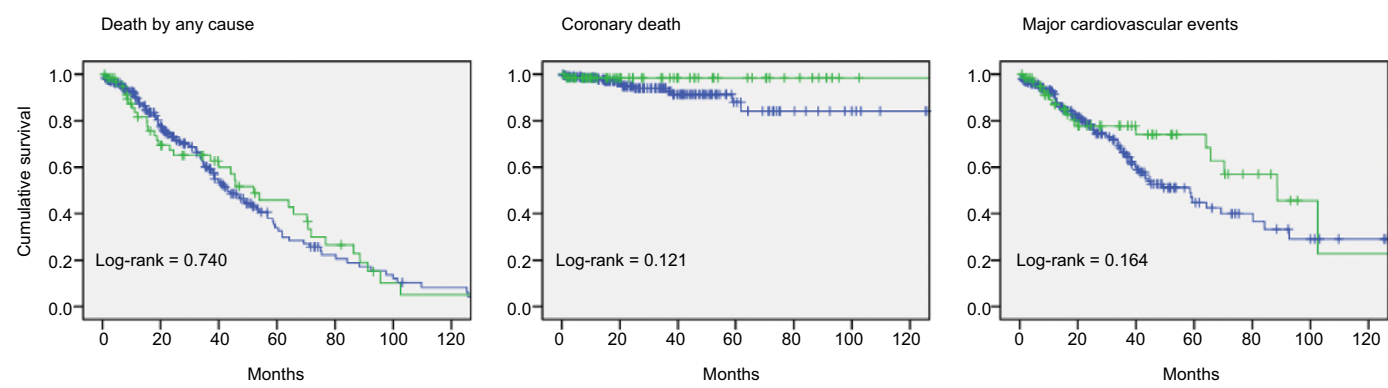

- Patients who underwent coronary angiography $n=213$

- Low-risk patients who did not undergo coronary angiography $n=63$

Figure 2 Impact of patient selection for coronary angiography on the incidence of death by any cause, coronary death, and major cardiovascular events in elderly hemodialysis patients.

Death by any cause

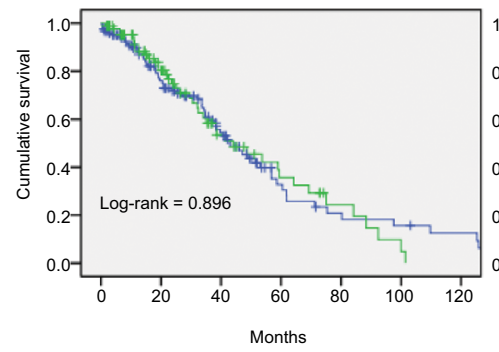

- Patients with coronary stenosis $\geq 70 \% n=124$

- Patients with normal arteries/nonsignificant coronary lesions $n=89$
Coronary death

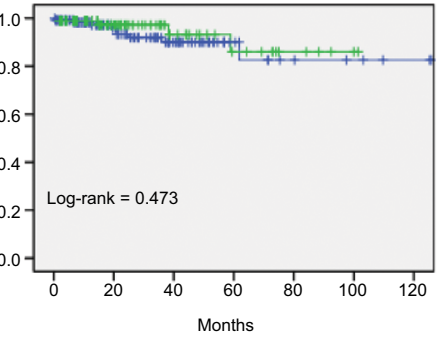$$
\text { (1) }
$$

Major cardiovascular events

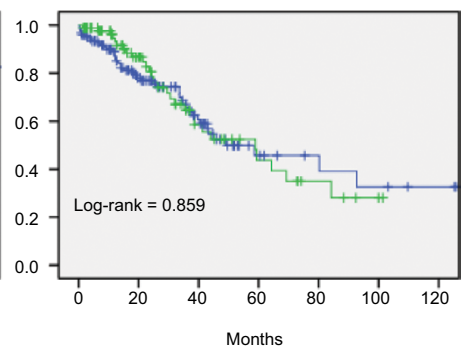

Figure 3 Impact of coronary artery disease defined by angiography on the incidence of death by any cause, coronary death, and major cardiovascular events in elderly hemodialysis patients.

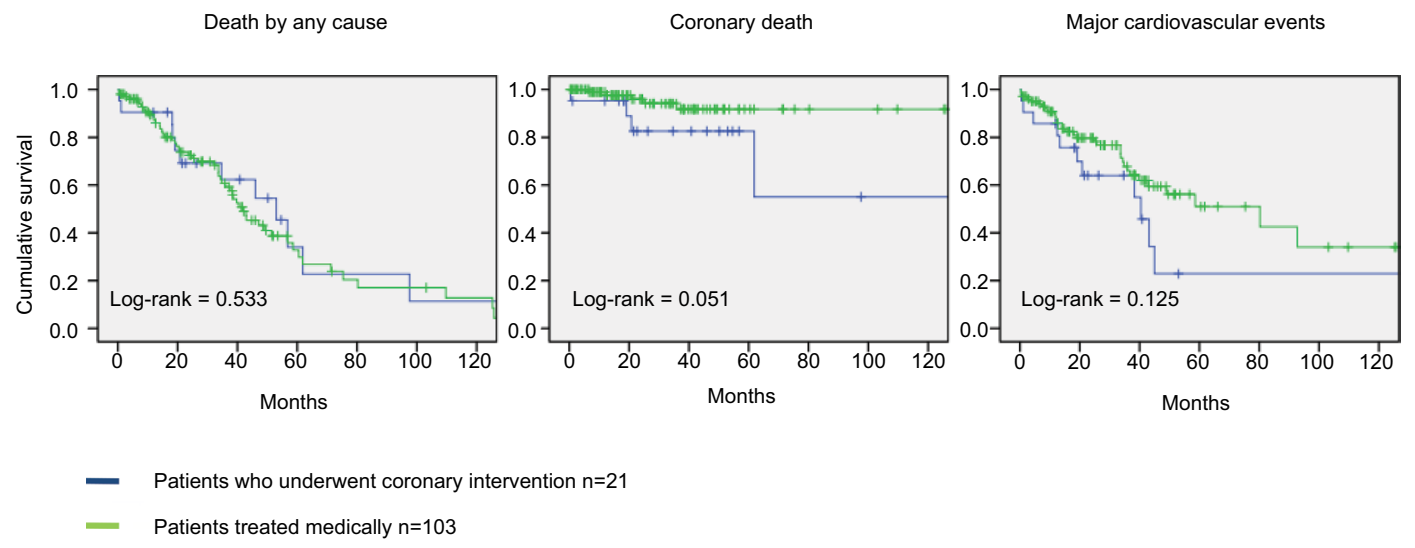

Figure 4 Impact of coronary intervention on prognosis in elderly hemodialysis patients.

patients underwent coronary artery bypass graft (CABG), and 13 were referred for percutaneous coronary intervention (PCI). The prevalence of multivessel disease was $85 \%$ in patients undergoing $\mathrm{CABG}$ and $63 \%$ in those undergoing PCI. Two subjects who underwent surgery died during the postoperative period, and no significant complications occurred with PCI.
In Figure 5, we compare the incidence of death by any cause in the four groups of patients shown at the bottom in Figure 1. No difference was found in prognosis in low-risk patients not selected for angiography, patients with normal or nonsignificant stenosis, those with significant stenosis treated medically, and those who had undergone coronary intervention. 
Death by any cause

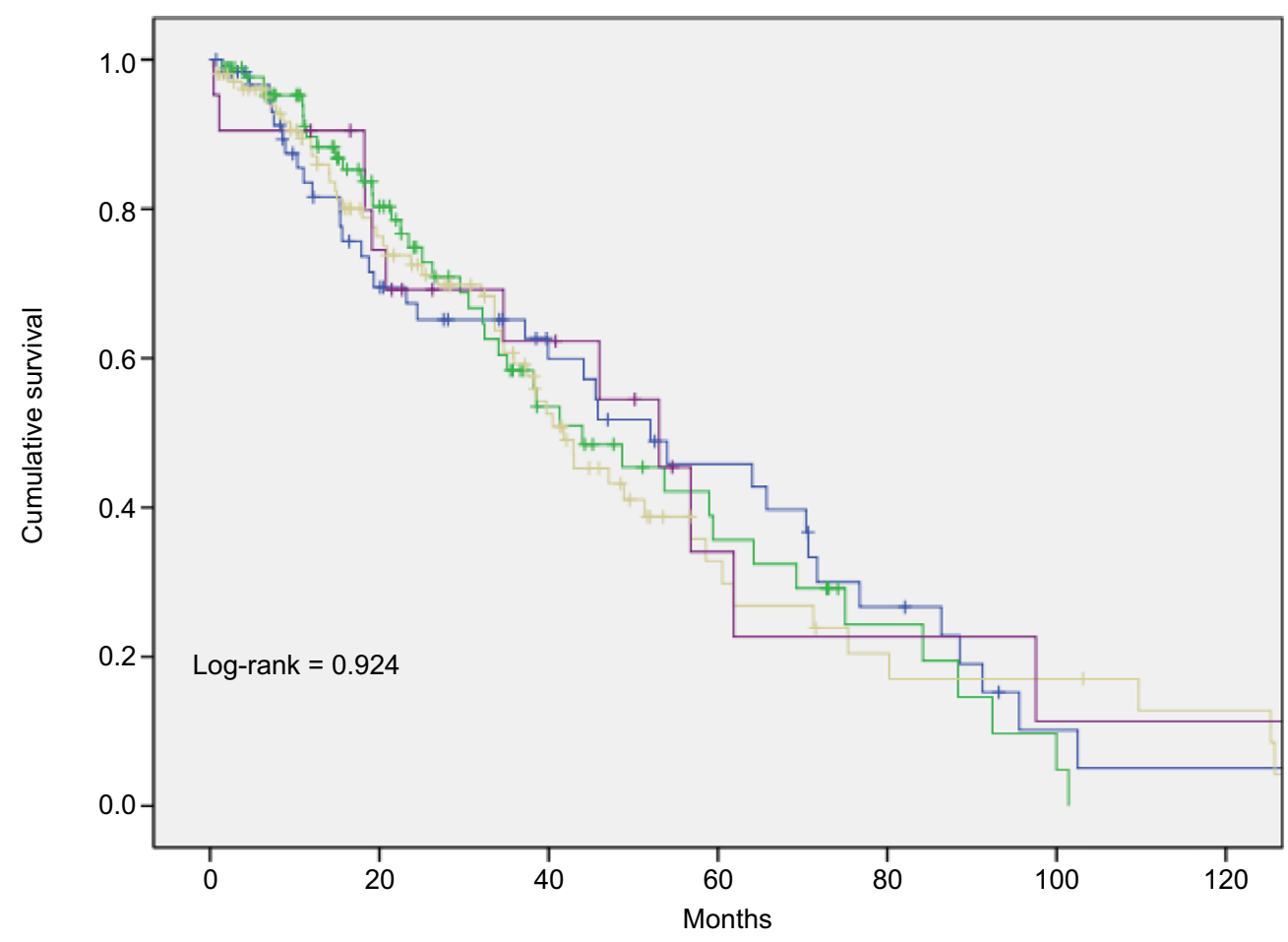

Low-risk, no coronary angiography, $n=63$

- Normal coronaries or nonsignificant stenosis, $n=89$

- Coronary stenosis $\geq 70 \%$, medical treatment, $n=103$

- Coronary stenosis $\geq 70 \%$, intervention, $n=21$

Figure $\mathbf{5}$ Coronary assessment and risk of death by any cause in elderly hemodialysis patients.

\section{Factors associated with the primary end point}

Table 3 shows the results of the multivariate Cox model that included age, sex, body mass index, hypertension, dyslipidemia, current/past smoking, diabetes, other CV diseases, and coronary assessment. Female sex (HR 1.676, 95\% CI $1.131-2.848, P=0.012$ ) was independently associated with death by any cause. Diabetes fell short of achieving statistical significance. Selecting patients for invasive investigation, based on clinical grounds, did not predict outcome $(95 \% \mathrm{CI}$ $0.636-1.695, P=0.944)$.

\section{Discussion}

In 1997, we started a prespecified protocol with the objective of defining the best approach to diagnose and treat cardiovascular disease in patients on the waiting list for renal transplantation, with emphasis on $\mathrm{CAD}$, using a
Table 3 Variables associated with primary end point (all-cause mortality)

\begin{tabular}{|l|l|l|l|}
\hline Variable & HR & $\mathbf{9 5 \%} \mathbf{C l}$ & P-value \\
\hline Age, years & $\mathrm{I} .015$ & $0.967-\mathrm{I} .067$ & 0.544 \\
\hline Sex, female & 1.676 & $\mathrm{I} .13 \mathrm{I}-2.484$ & 0.012 \\
\hline Body mass index, g/m $\mathrm{m}^{2}$ & 0.416 & $0.947-\mathrm{I} .023$ & 0.229 \\
\hline Hypertension & 0.07 & $0.674-1.098$ & $0.13 \mathrm{I}$ \\
\hline Dyslipidemia & 0.804 & $0.543-\mathrm{I} .189$ & 0.275 \\
\hline Smoking & $\mathrm{I} .334$ & $0.904-2.08 \mathrm{I}$ & 0.138 \\
\hline Diabetes & $\mathrm{I} .382$ & $0.962-1.985$ & 0.080 \\
\hline Other CV diseases* & $\mathrm{I} .378$ & $0.924-2.054$ & 0.116 \\
\hline Coronary assessment & $\mathrm{I} .017$ & $0.636-1.695$ & 0.944 \\
\hline
\end{tabular}

Notes: *Stroke, myocardial infarction, LV dysfunction, peripheral vascular disease. Abbreviations: CV; LV, left ventricular.

combination of clinical parameters and invasive and noninvasive coronary studies. We observed that this approach worked well for consecutive renal transplant candidates, in that patients selected for invasive coronary investigation 
had a poorer prognosis independently of the results of angiography. ${ }^{10}$

The purpose of this work was to assess the results of the same protocol for the evaluation of cardiovascular risk and death in elderly hemodialysis patients without angina. The design of the study precludes any conclusions on cause-effect relations, but the results are useful in informing clinicians on what can be expected when using protocols, based on clinical grounds and on the results of myocardial scan, for selecting aged CKD patients for coronary investigation and intervention.

An important premise behind screening for CAD in CKD patients is that the prevalence of CAD is high and is associated with a higher risk of events. However, there is no clear evidence that coronary assessment, based on that premise, will predict outcome in aged asymptomatic patients with advanced CKD.

In this investigation, we found that the prevalence of CAD was high but that coronary assessment was not useful as a risk stratification tool in this particular population of elderly subjects. We observed that neither patients selected for coronary investigation nor the actual presence of significant coronary disease predicted prognosis. Moreover, female sex, but not performing coronary assessment, was the independent predictor of the primary end point. Also important is the fact that, in spite of the high prevalence of CAD, coronary deaths were only 14 out of 143 deaths observed in the totality of our patients. Therefore, the data suggest that screening for CAD, with the protocol presented here, is not useful to identify asymptomatic aged patients including those at higher risk of events and should not be considered routine.

In agreement with our results, Aalten et $\mathrm{al}^{12}$ observed that coronary assessment had no impact in perioperative events in asymptomatic high-risk renal transplant patients and that only a small proportion of individuals with significant CAD underwent coronary intervention, as indicated by current guidelines.

On the other hand, the present results differ from those reported by our group of 519 consecutive patients from the same cohort that included young and elderly subjects (aged from 18 to 80 years) evaluated and treated using the same protocol. ${ }^{13}$ In that work, we found that CAD was associated with a threefold increase in cardiovascular events and with a 1.5-fold increase in the risk of all-cause mortality. In the general population, the COURAGE trial also showed that in patients treated with optimal medical therapy the atherosclerotic anatomic burden, as assessed by angiography, was a predictor of death, myocardial infarction, or non-ST segment elevation acute coronary syndrome. ${ }^{14}$

Because this was not a randomized study, the impact of coronary intervention on prognosis cannot be determined. However, the usefulness of a protocol based on current guidelines to select patients for intervention may be assessed. We found that using the American Heart Association and American College of Cardiology guidelines, ${ }^{8}$ coronary intervention was indicated in only $21 / 213$ patients undergoing coronary angiography $(9.8 \%)$, further reducing the relevance of coronary assessment. The low number of patients referred for intervention may be explained by the strict criteria, based on international guidelines, to indicate invasive procedures in that high-risk population; also, whenever PCI or CABG was considered possible by the guidelines, we gave preference to the former because of the higher risk of complications associated with surgery.

It can be argued that given the retrospective nature of the study, we cannot rule out the possibility that patients referred for intervention would fare worse if the intervention had not been undertaken, considering that patients eligible for CABG/ PCI had more severe CAD. In other words, the survival of patients with more severe CAD may have been improved by intervention to the level observed in patients treated medically. This issue should be addressed in a prospective, randomized trial including a larger number of individuals in whom both modalities of treatment are acceptable. However, even if that were the case, the low percentage of subjects selected for intervention would reduce any possible positive impact of investigation and intervention on prognosis.

The questionable benefit of coronary intervention in our patients departs from observations in older patients in the general population which showed that invasive treatment of CAD in the elderly is not harmful and may reduce mortality compared with medical therapy. ${ }^{15-17}$

In general, in patients with multivessel disease, $\mathrm{CABG}$ is considered the preferred modality of treatment, including in patients with end-stage renal disease. ${ }^{18,19}$ Bechtel et al analyzed the survival rates of patients on dialysis having bypass surgery during a 14 -year period. ${ }^{20}$ They showed that the 30-day mortality observed in nine German centers declined significantly from $27.7 \%$ in $1989-1993$ to $7.1 \%$ in 2000-2003, but the overall probability of survival did not change during the study period. Therefore, it seems that for patients with advanced $\mathrm{CKD}$, the observed improvements in the perioperative survival rates do not necessarily translate into an improved long-term prognosis. It must be stressed 
that the small number of patients undergoing intervention in our study prevents any definitive conclusion on that issue.

The reason for the apparent reduced impact of coronary investigation in the population of elderly subjects on dialysis may be related to their reduced life expectancy. It has been shown that patients aged 65 years and older treated by dialysis have a life expectancy of $\sim 4$ years, much lower than life expectancy for individuals of the same age without renal disease or younger subjects on dialysis. ${ }^{2}$ In patients older than 74 years on dialysis, the actuarial survival is even shorter, not higher than 3 years. ${ }^{21}$ Less than $20 \%$ of our patients were alive beyond 10 years of follow-up. It follows that older patients may not survive long enough to benefit from the diagnosis of CAD. They may have an increased prevalence of CAD but die early from other causes, as suggested by the small number of coronary deaths in our study. Also, it is possible that intensive medical treatment had a positive effect on prognosis independent of CAD. As we have learned from the past, this is a dynamic situation, and it is conceivable that in the future the implications of our work will be relevant for patients much older than 65 years.

\section{Conclusion}

In conclusion, in older asymptomatic patients on renal replacement therapy, the prevalence of CAD is high, but coronary investigation may not identify patients at higher risk of events. Therefore, in the elderly, coronary assessment should not be considered routine, at least in subjects without angina. The preferable interventional strategy for older patients on dialysis needs urgent investigation.

This work has limitations that must be addressed. This was a single-center retrospective study, and the number of patients was small, especially the individuals undergoing coronary intervention. Only bare metal stents were used. The number of patients withdrawn from the list could not be determined. Because all subjects were being considered for transplantation, the conclusions may not be valid for the totality of patients on renal replacement therapy and for patients not yet on dialysis. The choice for performing medical or surgical treatments in patients with CAD was decided on clinical grounds precluding any definitive conclusion on the best treatment modality. However, until prospective studies are available, that is what could be expected when applying current protocols to gauge our investigational and therapeutic choices.

\section{Disclosure}

The authors report no conflicts of interest in this work.

\section{References}

1. Jassal SV, Watson D. Dialysis in late life: benefit or burden. Clin J Am Soc Nephrol. 2009;4(12):2008-2012.

2. Canaud B, Tong L, Tentori F, et al. Clinical practices and outcomes in elderly hemodialysis patients: results from the Dialysis Outcomes and Practice Patterns Study (DOPPS). Clin J Am Soc Nephrol. 2011;6(7): 1651-1662.

3. Charytan D, Kuntz RE. The exclusion of patients with chronic kidney disease from clinical trials in coronary artery disease. Kidney Int. 2006;70(11):2021-2030.

4. Collins AJ, Foley RN, Herzog C, et al. USRDS 2012 Annual Data Report. Am J Kidney Dis. 2013;61(1):e1-e421.

5. Galvão de Lima JJ, Wolff Gowdak LH, de Paula FJ, et al. The role of myocardial scintigraphy in the assessment of cardiovascular risk in patients with end-stage chronic kidney disease on the waiting list for renal transplantation. Nephrol Dial Transplant. 2012;27(7): 2979-2984.

6. De Lima JJ, Sabbaga E, Vieira ML, et al. Coronary angiography is the best predictor of events in renal transplant candidates compared with noninvasive testing. Hypertension. 2003;42(3):263-268.

7. Lentine KL, Costa SP, Weir MR, et al. Cardiac disease evaluation and management among kidney and liver transplantation candidates. $J \mathrm{Am}$ Coll Cardiol. 2012;60(5):434-480.

8. Patel MR, Dehmer GJ, Hirshfeld JW, et al. ACCF/SCAI/STS/AATS/ AHA/ASNC 2009 Appropriateness Criteria for Coronary Revascularization: a report by the American College of Cardiology Foundation Appropriateness Criteria Task Force, Society for Cardiovascular Angiography and Interventions, Society of Thoracic Surgeons, American Association for Thoracic Surgery, American Heart Association, and the American Society of Nuclear Cardiology Endorsed by the American Society of Echocardiography, the Heart Failure Society of America, and the Society of Cardiovascular Computed Tomography. J Am Coll Cardiol. 2009;53(6):530-553.

9. Poldermans D, Schouten O, Vidakovic R, et al. A clinical randomized trial to evaluate the safety of a noninvasive approach in high-risk patients undergoing major vascular surgery: the DECREASE-V Pilot Study. J Am Coll Cardiol. 2007;49(17):1763-1769.

10. De Lima JJ, Gowdak LH, de Paula FJ, Muela HC, David-Neto E, Bortolotto LA. Coronary artery disease assessment and intervention in renal transplant patients: analysis from the KiHeart cohort. Transplantation. 2016;100(7):1580-1587.

11. Smith SC, Benjamin EJ, Bonow RO, et al. AHA/ACCF Secondary Prevention and Risk Reduction Therapy for Patients with Coronary and other Atherosclerotic Vascular Disease: 2011 update: a guideline from the American Heart Association and American College of Cardiology Foundation. Circulation. 2011;124(22):2458-2473.

12. Aalten J, Peeters SA, van der Vlugt MJ, Hoitsma AJ. Is standardized cardiac assessment of asymptomatic high-risk renal transplant candidates beneficial? Nephrol Dial Transplant. 2011;26(9): 3006-3012.

13. De Lima JJ, Gowdak LH, de Paula FJ, et al. Treatment of coronary artery disease in hemodialysis patients evaluated for transplant-a registry study. Transplantation. 2010;89(7):845-850.

14. Mancini JBG, Hartigan PM, Shaw LJ. Predicting outcome in the COURAGE trial. J Am Coll Cardiol Intv. 2014;7:195-201.

15. The Bypass Angioplasty Revascularization Investigation (BARI) Investigators. Comparison of coronary bypass surgery with angioplasty in patients with multivessel disease. $N$ Engl J Med. 1996;335: 217-225.

16. TIME Investigators. Trial of invasive versus medical therapy in elderly patients with chronic symptomatic coronary-artery disease (TIME): a randomised trial. Lancet. 2001;358(9286):951-957.

17. Graham MM, Norris CM, Galbraith PD, Knudtson ML, Ghali WA; APPROACH Investigators. Quality of life after coronary revascularization in the elderly. Eur Heart J. 2006;27(14):1690-1698. 
18. Sipahi I, Akay MH, Dagdelen S, Blitz A, Alhan C. Coronary artery bypass grafting vs percutaneous coronary intervention and long-term mortality and morbidity in multivessel disease: meta-analysis of randomized clinical trials of the arterial grafting and stenting era. JAMA Intern Med. 2014;174(2):223-230.

19. Marui A, Kimura T, Nishiwaki N, et al. Percutaneous coronary intervention versus coronary artery bypass grafting in patients with end-stage renal disease requiring dialysis (5-year outcomes of the CREDO-Kyoto PCI/CABG Registry Cohort-2). Am J Cardiol. 2014;114(4):555-561.
20. Bechtel JF, Detter C, Fischlein T, et al. Cardiac surgery in patients on dialysis: decreased 30-day mortality, unchanged overall survival. Ann Thorac Surg. 2008;85(1):147-153.

21. Panduranga R, Robinson B, Schaubel DE. Mortality. In: 2016 Annual Data Report (ADR) of the United States Renal Data System (USRDS) Volume 2: End-Stage Renal Disease in the United States. Available from: https://www.usrds.org/2016/view/v2_06.aspx. Accessed January 22, 2018.
The International Journal of Nephrology and Renovascular Disease is an international, peer-reviewed open access journal focusing on the pathophysiology of the kidney and vascular supply. Epidemiology, screening, diagnosis, and treatment interventions are covered as well as basic science, biochemical and immunological studies. The manuscript management system is completely online and includes a very quick and fair peer-review system, which is all easy to use. Visit http://www. dovepress.com/testimonials.php to read real quotes from published authors.

Submit your manuscript here: https://www.dovepress.com/international-journal-of-nephrology-and-renovascular-disease-journal 